from Lander's tale is that the new technique must be used with care. When has it ever been otherwise with a new technology?

Many of the difficulties are those arising whenever people seek to turn a research technique into a routine assay - techniques borrowed from cytological research for the early diagnosis of cervical cancer, for example, or from strength-of-materials engineering for assessing liability to metal fatigue. In research laboratories, where the objective is usually to test original hypotheses about the natural world, people quite properly adjust the characteristics of their experimental systems to provide the most stringent tests of their hypotheses. Routine assays, by contrast, are best standardized, not merely for convenience, but so that experience will assist empirical understanding of their usefulness and limitations. That is bound to take time.

Lander's article points to several respects in which DNA fingerprinting must be applied with caution, among which the most interesting yet teasing is the demonstration (by an examination of blood-bank samples) that polymorphic alleles are not scattered at random through real human populations. That is hardly a surprise; intermarriage between ethnic, cultural or even social groups is also far from random. So attempts accurately to calculate the chance that two apparently identical samples of DNA are from different individuals require not only dependable laboratory techniques, but also a knowledge of the distribution of the target alleles among the sub-populations from which the people concerned are drawn. This is a potentially daunting obstacle to the calculation of odds sufficiently astronomical to impress trial juries, however great may be its promise for human anthropologists.

How, meanwhile, should the forensic use of DNA fingerprinting be encouraged without the technique being oversold? The first need is that users should not expect too much. In principle, measurement of the lengths of restriction fragments of people's DNA is no different from the use for forensic purposes of more familiar genetic markers - the $\mathrm{A}, \mathrm{B}$ and $\mathrm{O}$ blood groups, for example. Genetic differences between individuals can be established unambiguously, but there is always a chance that an appearance of genetic identity may be a coincidence. The promise of DNA fingerprinting, still unchallenged, is that the chances of accidental genetic matches will be very much smaller. The confusion at the pre-trial hearings in New York centres on more practical questions, most of them about the need for standard laboratory procedures. (Given that people's liberty is at stake, there is also a need for rules on the custody and use of samples.) If the US National Academy of Sciences believes it could help, it should carry out the study being urged on it without waiting for a subsidy.

Meanwhile, there is a need that everybody should be on guard against the seductiveness of large numbers. When the world's population is of the order of $10^{11}$, it cannot much matter that the chance of an accidental genetic match is given as one in $10^{12}$ or one in $10^{15}$. In most circum- stances, a few extra polymorphic sites could always be used to add a few orders of magnitude to the odds. What juries (and the rest of us) need to understand is that there are exceptions to the sense of safety deriving from these very large numbers - the case of identical twins most obviously, but those arising from flagrant departures from Hardy-Weinberg equilibrium will be more taxing. DNA fingerprinting has a valuable contribution to make to forensic science and the administration of justice, but its evidence, like most other evidence, requires interpretation. (The practical application of gene amplification techniques, which in principle offer identification by a single molecule of DNA, will be similarly beset with difficulties.)

The underlying difficulty is that science and the law differ in temperament. Both pretend to discover truth, science by continually narrowing the range of allowable dispute, the law by its supposition that the judicial process can conjure truth from whatever data are available. Courts are not content with declarations that defendants are, say, "60 per cent guilty", nor should they be.

\section{When to track AIDS}

New York City authorities ask whether the time has come to trace the contacts of AIDS patients. It has not.

Curious though it may be, it is also heartening that the illiberal notions prevalent five years ago for dealing with the then impending spread of AIDS appear to have melted away. Much of the explanation lies in the characteristics of the infection as now recognized: the long and variable period before antibodies make their appearance (allowing for diagnosis) and the long and variable incubation period before the onset of the overt disease, plainly argue against equitable, let alone effective, ostracization. So far, even the most seriously affected communities have resisted even tracing the sexual or needle-sharing partners of those with AIDS on the proper grounds that, in the absence of prophylaxis, knowing who is liable to be struck down would be of little use.

It is natural that the Health Commissioners for New York City should have been (last week, at Montreal see page 496) the first to call for measures to enforce contact-tracing. The scale of New York's AIDS pandemic is daunting, while there is now some hope that AIDSassociated pneumonia can be prevented by drugs. But the time for compulsory contact-tracing has not yet arrived. First, the efficacy of the prophylaxsis needs more secure demonstration. Second, the prospect that contacts would be traced is likely more effectively to dissuade people from seeking treatment than with the simpler (and tractable) venereal diseases. But there remains no assurance that contact-tracing for AIDS, with its inevitable loss of confidentiality, could be undertaken without the pointlessly discriminatory reprisals against the infected that even enlightened communities condone. 